\title{
Dilemas éticos en los servicios sociales de base de la Mancomunidad de Sakana
}

\author{
Nahia Urquía Ihabar \\ Departamento de Trabajo Social, Universidad Pública de Navarra
}

\section{Francisco Idareta-Goldaracena}

Departamento de Trabajo Social, Universidad Pública de Navarra

francisco.idareta@unavarra.es

\begin{abstract}
Gizarte Laneko diziplinan, esku-hartze sozialak konpromezu etikoa beharrezkoa du une oro pertsona erabiltzaileen eskubide urraketak ez emateko, baina oso gutxi dira landa-eremuetako Gizarte Zerbitzuetan etikari buruz eginiko ikerketak. Horregatik, artikulu honek profesional hauek bizi duten errealitatea ezagutu nahi izan du. Lortutako emaitza profesionalek darabilten teoriaren eta praxiaren arteko eten handia islatzen dute; teorizatzeko joera baitago, baina ez joera hori praktikara eramatekoa. Era berean, inkongruentzia aurkitzen da profesionalek azaltzen dutenaren eta benetan egiten denaren artean. Esku-hartzean etika oso garrantzitsua dela adierazten dute profesionalek, baina errealitatean intuizioz, gogoeta gehiegirik gabe eta etika profesionalarekin zerikusia duten alderdi beharrezkoei arreta jarri gabe dihardute.
\end{abstract}

\section{GAKO-HITZAK:}

Dilema etikoak, Gizarte Langintza, esku-hartze soziala, landa-eremua, prestakuntza etikoa.
El compromiso ético de las y los profesionales de los servicios sociales es fundamental para evitar la vulneración de los derechos de las personas usuarias, pero apenas hay estudios sobre ética en los servicios sociales en el ámbito rural. Por ello, este artículo pretende aproximarse a la realidad de estos profesionales de lo social. Los resultados obtenidos reflejan una gran brecha entre la teoría que manejan las profesionales y la praxis, ya que se tiende a teorizar demasiado pero no a aplicarlo en la práctica. Al mismo tiempo, se encuentra una incongruencia entre lo que explican las y los profesionales y lo que realmente hacen, pues aseguran que la ética es muy importante en la intervención, pero en la práctica actúan principalmente por intuición, sin reflexionar suficientemente y sin prestar la atención necesaria a los aspectos relacionados con la ética profesional.

\section{Palabras Clave:}

Dilemas éticos, trabajo social, intervención social, zona rural, formación ética. 


\section{Introducción}

El presente artículo tiene como finalidad aproximarnos a la conciencia ética que tienen las y los profesionales de los servicios sociales de base de la Mancomunidad de Sakana (Navarra). Esto se debe a que los dilemas éticos son una realidad del día a día de las y los profesionales, aunque su identificación en la práctica, como veremos, sea más difícil. Más aún si tomamos como punto de partida que los servicios estudiados en este trabajo se encuentran en una zona rural con mayores limitaciones de recursos que la zona urbana.

Por ello, el objetivo de este estudio consiste en comprobar si las y los profesionales de los servicios sociales de base de Sakana son conscientes de los dilemas éticos que se dan en la intervención social y cuáles son los que identifican. Nos encontramos, pues, ante una disciplina que establece una relación vinculante con la realidad y requiere de una reflexión ética. Todo ello basándonos en el estudio realizado en la Comunidad Foral de Navarra por Úriz, Ballestero y Urien (2007).

En la actualidad, las y los profesionales se enfrentan a numerosos dilemas éticos en la intervención social. Los cambios acaecidos en los últimos años han afectado considerablemente a los distintos ámbitos en los que se ejerce la intervención social, complejizándose de tal manera que, consecuentemente, dan lugar a nuevos dilemas éticos (Ballestero, Úriz y Viscarret, 2012).

Así lo corrobora el Consejo General del Trabajo Social (CGTS, 2012). En las memorias publicadas en el año 2012, ya comenzó a observarse un aumento de consultas realizadas por las y los profesionales colegiados españoles. En ellas, las principales cuestiones estaban relacionadas con lo laboral, la interpretación de normativas, derechos, etc.

El acceso a nuevas funciones y al desempeño de nuevos roles de las y los profesionales es, en gran medida, consecuencia de los nuevos fenómenos y demandas sociales que han surgido en estos tiempos (Ballestero, Úriz y Viscarret, 2012). En España se ha dado un incremento de nuevas necesidades sociales surgidas como consecuencia del proceso de modernización y la crisis, donde se aumenta la presencia de las y los trabajadores sociales en nuevos y diferentes ámbitos profesionales (Ballestero, Úriz y Viscarret, 2012).

Fruto de esta diversidad y de los cambios de nuestra sociedad, se han determinado distintas áreas de actuación de las y los profesionales en diferentes ámbitos: "servicios sociales polivalentes y especializados, servicios educativos, servicios de salud, servicios relacionados con la administración de justicia, servicios relacionados con el sector laboral, con el sector vivienda, ejercicio privado, administración, gerencia y planificación de servicios de bienestar, y docencia e investigación" (Domínguez, 2005: 78).
Como consecuencia de ello, la praxis profesional se convierte en tan diferente y diversificada como los ámbitos en los que se desarrolla el trabajo social (Hernández, 2003). De tal manera que la intervención realizada tanto desde la atención primaria como especializada estén abarcando casos multiproblemáticos de "infancia, adolescencia, jóvenes, mujeres, personas mayores, personas con discapacidades, minorías étnicas, familias, enfermos, presos y exreclusos, extranjeros y cualquier otro que por su situación de necesidad o problema lo requiera" (Domínguez, 2005: 78).

No obstante, como señalan Nelson, Pomerantz y Weeks (2006), nos encontramos ante un área poco investigada a nivel nacional e internacional. Y lo mismo sucede en la Comunidad Foral de Navarra, donde la escasísima bibliografía hallada acerca del tema parece no tener en cuenta más que a la zona urbana. A nivel internacional, gran parte de la literatura escrita sobre ética hasta el siglo XXI se encuentra recogida en el ámbito anglosajón, aunque en los últimos años se haya publicado mucho más, mayoritariamente desde el ámbito de la salud (Roberts, Battaglia y Epstein, 1999; Roberts, Battaglia y Epstein, 1999; Nelson, Greene y West, 2010; Nelson, Pomerantz y Weeks, 2006).

Estas nuevas investigaciones relatan una realidad parecida a la estudiada en este trabajo. La ubicación del estudio se encuentra en un área alejada, de poca densidad poblacional, con escasez de recursos y donde la importancia del vínculo entre las y los profesionales y las personas usuarias es muy importante. En este sentido, un factor a subrayar es la confianza mutua y el conocimiento que tienen las personas que viven en estos entornos, donde sus relaciones son estructuradas por los códigos culturales compartidos a través de su historia. De ahí que la simbología rural deba ser comprendida y compartida por las y los profesionales para poder llevar a cabo una intervención cercana y profesional.

A todo lo anterior se suman los cambios territoriales en materia de competencias políticas, que condicionan los distintos tipos de dilemas éticos. Lo mismo ocurre con la situación orográfica de Navarra: montañosa al norte y llana al sur, ambas con municipios pequeños y alejados de las ciudades donde gozan de la mayoría de servicios. En este aspecto, el valle de Sakana, al noroeste de la Comunidad Foral de Navarra, se caracteriza por ser una agrupación de pequeños y alejados municipios que está definida como zona rural.

Como señala García (1987), en la zona rural se concentra menor densidad poblacional y hay un contacto más estrecho y directo, aunque a nivel institucional exista una mayor limitación material y los servicios sean insuficientes en la mayoría de los casos. Esto dificulta el normal desarrollo de las actividades que, como más adelante señalaremos, es uno de los motivos que nos ha llevado a realizar el presente estudio. 
Aunque la novedad en la investigación no siempre es garantía de éxito, sí que parece que es síntoma de progreso en el campo correspondiente, permitiendo ofrecer a los interesados (profesionales, académicos, estudiantes, docentes, etc.) nuevos datos y puntos de vista sobre uno de los ámbitos de la profesión tan interesante como poco investigado (Úriz, Ballestero y Urien, 2007).

A la vista de todo ello, es necesario incidir en la ética profesional, ya que parte importante de esta ética es la que se ocupa de desarrollar valores y principios que han de servir de fundamento a cada profesión (Úriz, Ballestero y Urien, 2007; Idareta, Úriz y Viscarret, 2017). Así pues, el estudio se estructura en cuatro apartados. En el primer apartado, se realiza un breve acercamiento al tema de estudio. En el segundo apartado, se explican las diferentes metodologías utilizadas para realizar el trabajo. En tercer lugar, se explican los resultados obtenidos a través de tales metodologías. Por último, el trabajo finaliza exponiendo las conclusiones obtenidas y presentando las propuestas recogidas por las y los profesionales.

Con todo ello, se identifican algunas situaciones éticamente problemáticas y se ofrecen algunas pautas básicas para su resolución.

\section{2. Ética en los servicios sociales del ámbito rural}

Para poder analizar la dimensión ética en los servicios sociales de base de Sakana debemos entender las caracteristicas propias de la zona rural, pues es una de las áreas menos investigadas desde la perspectiva ética.

Como ya se ha adelantado, no son muchas las públicaciones realizadas en este área de intervención. Principalmente son publicaciones internacionales las que estudian la realidad rural, pero con particularidades propias. Canadá, Botswana, Estados Unidos, Nuevo México y Alaska, son algunos de los lugares donde se han realizado estas investigaciones por autores como Mackie (2007), Roberts, Warner y Hammond, (2005), Pesut, Bottorff, y Robinson (2011), Nelson, Pomerantz y Weeks (2006), Nelson, Greene y West (2010) y Akinsola (2001).

De entre los estudios a nivel nacional y los cuestionarios sobre las funciones de las y los profesionales a nivel regional, destaca el realizado por Úriz, Ballestero y Urien (2007). En este sentido, en materia de ética e intervención social en los servicios sociales de base de Navarra, solo encontramos los trabajos realizados por el grupo de investigación Efimec (Ética, Filosofía y Metodología de la Ciencia) de la Universidad Pública de Navarra.

Efectivamente, tanto los valores como los principios son fundamentales en la intervención social. Los valores son criterios que utilizamos para seleccionar determinadas conductas que consideramos buenas 0 deseables, según cada teoría ética, y en la disciplina de trabajo social hacen referencia a una serie de principios morales/éticos fundamentales con los cuales se "comprometen o deberían comprometerse los trabajadores sociales" (Banks, 1997: 8). Estos principios quedan recogidos en las declaraciones y convenciones internacionales de derechos humanos, las cuales estructuran las pautas internacionales, y reconocen los derechos aceptados por la comunidad internacional.

En las siguientes líneas señalamos algunas de las herramientas especialmente relevantes para la buena praxis en el ejercicio del trabajo social. Entre ellas se encuentran la Declaración Universal de Derechos Humanos, el Pacto Internacional sobre Derechos Civiles y Políticos, el Pacto Internacional sobre Derechos Económicos, Sociales y Culturales, la Convención para la Eliminación de todas las Formas de Discriminación Racial, la Convención para la Eliminación de todas las formas de Discriminación contra las Mujeres, la Convención sobre los Derechos del Niño y la Convención sobre Pueblos Indígenas y Tribales -convención de la OIT 169- (Aranguren, Peña y Villaño, 2008).

Los valores y principios éticos que guían la relación profesional son pilares básicos sobre los que se asienta la intervención social. En este sentido, los dilemas éticos se estudian desde la dimensión pragmática (Bermejo, 2002) y han sido definidos por Banks (1997) como situaciones de toma de decisiones que conllevan la difícil elección entre dos o más alternativas no deseables o donde no es clara la elección. Por su parte, Ballestero, Viscarret y Úriz (2012) señalan que los dilemas éticos aparecen cuando hay un conflicto entre principios que conducen a cursos de acción excluyentes. En las últimas décadas, como señalan Cortina y Conill (2000), existe la necesidad de desarrollar más profundamente la llamada “ética profesional”. Y, aunque las teorías éticas orientan nuestra práctica profesional, muy pocos profesionales son capaces de identificarlas (Idareta, 2013). Consecuentemente, se reitera la necesidad de reflexionar sobre la intervención social desde una perspectiva ética, tomando conciencia de los diferentes dilemas éticos a los que las y los profesionales se enfrentan. Más aún en el contexto rural, donde, por las propias caracteristicas de la zona, la relación entre profesionales y personas usuarias es más estrecha y continuada en el tiempo.

\section{Metodología}

El estudio se ubica en una zona rural de la Comunidad Foral de Navarra. En concreto, en el valle de Sakana, que se encuentra al noroeste de la región ${ }^{1}$. Los tres servicios sociales de base se

${ }^{1}$ El valle posee una extensión total de 306,884 km² y la población es de aproximadamente de unas 20.000 personas. 
sitúan en aquellos municipios con mayor densidad poblacional (Alsasua, Echarri-Aranaz e Irurzun) agrupando sus propios municipios con el fin de personalizar más la atención ${ }^{2}$. En ellos se llevan a cabo los programas de promoción de la autonomía personal y atención a personas en situación de dependencia, incorporación social, acogida y orientación y atención a familias, infancia y juventud.

Frente a esta realidad tan diversa y diferenciada, las formas en las que se presentan los dilemas éticos serán igualmente diversas y de múltiples tipologías. Por eso a las y los profesionales a menudo les surgen distintas cuestiones éticas ante las que cabe preguntarse cómo tomar adecuadamente una decisión sin dejar de proporcionar a la persona usuaria la mejor atención.

La muestra de la investigación concuerda con el universo debido al número de las y los trabajadores de lo social ${ }^{3}$ que desempeñan su labor en los servicios sociales de base de la Mancomunidad de Sakana: en Irurzun trabajan once profesionales, en Alsasua siete y en Echarri-Aranaz, cinco.

Al igual que en el estudio realizado por Úriz, Ballestero y Urien (2007), en los datos anteriores se observa que la dispersión de las y los trabajadores de lo social por la Mancomunidad de Sakana responde a la estructura de los servicios sociales de base que, a su vez, responde al binomio población-necesidades sociales.

Cuadro 1. Áreas tratadas en el cuestionario
Se utilizó metodología cuantitativa y cualitativa. En cuanto a la metodología cuantitativa, se adaptó un cuestionario que parte de los estudios realizados en Estados Unidos sobre el tema por la doctora Eileen Joan $\operatorname{Sin}(2001)^{4}$.

Al cuestionario se añadieron una carta explicativa sobre la voluntariedad en la participación, el consentimiento informado y un apartado para enviar las respuestas y poder garantizar la confidencialidad de los datos. El cuestionario original está dividido en cinco secciones: formación en ética, conocimiento y uso del código de ética, sistemas de apoyo ético, dilemas éticos percibidos y características demográficas. Para preservar la confidencialidad, se han omitido ítems a partir de los que se pudiese identificar a las personas que han respondido el cuestionario.

Para realizar esta investigación, se ha adaptado el cuestionario de Úriz, Ballestero y Urien (2007) a la realidad de la Mancomunidad de Sakana. Por eso se ha optado por reducir el estudio a las áreas que recoge el Cuadro 1.

Mediante la metodología cualitativa, se busca recopilar información sobre las estrategias profesionales de las y los trabajadores de lo social y cómo hacen frente en su quehacer diario a los dilemas éticos. Las entrevistas se han estructurado a partir de las preguntas que realizan en su estudio Úriz, Ballestero y Urien (2007), puesto que se parte

Datos personales
- Sexo.
- Edad.
- Títulos académicos
que posee.
- Experiencia
profesional como
trabajador de lo social.
- Su sueldo anual neto.

Datos profesionales
- Jornada de trabajo
en el servicio social
de base.
- Trabajo habitual que se
relaciona con las áreas
de intervención social.
- Problemas más
frecuentes que le toca
afrontar en el trabajo.

Dilemas éticos
- Conocimiento de los
dilemas éticos en función
del perfil profesional
desempeñado.
- Identificación de algún
dilema ético relacionado
con alguno de los temas
(confidencialidad,
autonomía del cliente,
abuso de poder, etc.) y su
incidencia.

Dilemas éticos en el trabajo diario
- Satisfacción sobre la resolución
de algún dilema ético.
- Estrategias de resolución de los
dilemas éticos.
- Apoyo judicial en la resolución
de los dilemas éticos.
- Precedentes legales
relacionados con algunas de las
decisiones éticas.

2 En el caso de Echarri-Aranaz, cuyo servicio agrupa menos municipios, encontramos que la provisión de servicios se dirige a los pueblos de Urdiain, Iturmendi, Bacaicoa, Echarri-Aranaz, Arbizu, Lacunza y Ergoiena (que se compone de Unanua, Torrano y Lizarraga). Consta de los programas de Atención a Infancia y Familia, Drogodependencia, Incorporación Social, Empleo protegido e Inmigración. Por su parte, Alsasua consta de los municipios de Ciordia, Olazagutía y Alsasua, donde se desarrollan los programas de Acogida, Empleo social, Prevención, Servicio de atención a domicilio y Psicología. Por último, encontramos Irurzun, el más amplio en cuanto a la dimensión geográfica, que agrupa los municipios de Lecumberri, Uharte Araquil, Echalecu, Araiz, Betelu y el propio Irurzun.

3 Profesionales de la intervención social (educadores sociales, psicólogos, trabajadores sociales etc.).
4 Este cuestionario fue revisado y aprobado por los doctores Philip J. Boyle, director adjunto del Hastings Institute (Vancouver, Canadá), vicepresidente y editor jefe de Park Ridge Centre (Chicago, Illinois); Jonathan D. Moreno, catedrático de Bioética de la Universidad de Virginia y director del Centro de Bioética; y Bárbara Ann Lieberman, experta en estadística y analista de programas del Ministerio de Sanidad y Servicios Sociales de Estados Unidos. 
del mismo estudio, de manera que pudieran servir para ampliar y enriquecer algunas respuestas obtenidas a partir de los cuestionarios.

\section{Análisis de resultados}

En primer lugar, se exponen los datos personales de las y los profesionales, donde se recoge su sexo, edad, titulaciones académicas y experiencia profesional. En segundo lugar, se plantean los datos profesionales, que se componen de la jornada laboral de las y los profesionales, el área donde intervienen, la tarea principal que desempeñan en las áreas, $y$, por último, el principal problema con el que se encuentran al intervenir. Finalmente, se analizan las entrevistas efectuadas.

\subsection{Datos personales}

En cuanto a los datos personales, y como se constata en los distintos estudios realizados hasta el momento, la tradición mayoritariamente femenina de la profesión se reitera en la actualidad, como refleja el estudio, con un $99 \%$ de mujeres profesionales. Esta tradición se debe al carácter de beneficencia que caracterizó a la profesión hasta hace poco (las jornadas de asistentes celebradas en Pamplona en 1977, en las que se tomó la decisión de cambiar el nombre de asistentes por el de trabajadores sociales), donde se reproducía en la esfera pública el papel que tradicionalmente desempeñaba la mujer en la esfera privada, así como a la particularidad de ser en sus inicios un trabajo voluntario para los demás, sin remuneración y realizado por mujeres, en un contexto donde el trabajo retribuido era esencialmente masculino.

El factor de la edad nos ofrece un sector profesional mediano, con experiencia suficiente, ya que la mayoría de las y los profesionales se encuentra entre los 41-50 años de edad. Al igual que en la investigación realizada por Úriz, Ballestero y Urien (2007), la explicación de los datos reside en que hasta 1984, en Navarra no existían estudios de Trabajo Social de nivel universitarios. Fue en el curso 1990-1991 cuando se empezó a impartir la diplomatura en la Universidad Pública de Navarra.

Asimismo, gran parte de las y los profesionales ha realizado un recorrido académico que se inicia en la formación profesional, acabando con la formación universitaria, por lo que los pasos de los años académicos inciden en la temporalidad de la inserción al mundo laboral, ya que al mismo tiempo que se instauran y desarrollan los estudios universitarios de Trabajo Social, se desarrollan los servicios sociales (entre 1984-1990) y con ellos se inician las convocatorias de oposiciones (Úriz, Ballestero y Urien, 2007)

La formación académica de las y los trabajadores de lo social está condicionada por la oferta de formación profesional y universitaria. Esto se debe a la exigencia administrativa que obliga a presentarse a las oposiciones con diplomatura. De igual manera, la ubicación geográfica de las y los profesionales, la mayoría proveniente del valle, limita su movilidad.

La mayoría de las y los profesionales que trabajan en los servicios cuentan con el diploma de Trabajo Social (un $25 \%$ ). La razón de que el cómputo mayoritario de los estudios resida en el ítem "otros" (35\%), se debe a que la mayoría de las personas entrevistadas ha realizado distintos estudios de formación profesional, así como diferentes cursos de expertos, todos ellos relacionados con el ámbito social en el que trabajan. Es de subrayar que un $15 \%$ de los profesionales haya cursado el diploma en Educación Social y que un $10 \%$ haya realizado la formación profesional de técnico en atención sociosanitaria, particularmente en los profesionales del área de prevención a la dependencia y promoción a la autonomía personal. El 12,5\% de los técnicos ha realizado el curso de experto en gestión de servicios sociales. Esto se debe a que en el año 2016-2017 se hizo una propuesta académica a los y las trabajadores de los servicios sociales de Navarra para la ampliación de la formación. Solo una de las personas menciona que no posee ninguna titulación relacionado con el Trabajo Social.

En cuanto a las universidades o instituciones educativas donde han realizado los estudios, se observa una realidad mayoritaria. El 55,2\% han realizado los estudios en la Univeridad Pública de Navarra, seguida de la Universidad del País Vasco $(20,7 \%)$. Esto quizás se deba a la cercanía geográfica que tienen ambas instituciones.

La experiencia media de las y los trabajadores de lo social de los servicios sociales de base de Sakana es de 13,05 años y responde a causas históricas, como fue el desarrollo de los servicios sociales de base a finales de los años ochenta. En los resultados vemos que un $46 \%$ de profesionales tienen una experiencia superior a diez años, lo cual concuerda con el análisis de la edad descrito con anterioridad.

\subsection{Datos profesionales}

La mayoría de las y los profesionales trabaja a jornada completa ( $84 \%$ ), un pequeño grupo a media jornada y un $4 \%$, a tres cuartos de jornada. Los últimos datos se clasifican también por la feminización de la profesión, que muchas veces lleva a las mujeres a reducir su jornada por la conciliación familiar. En el siguiente apartado se observa la multiplicidad de colectivos que abarca la profesión. El hecho de que en la zona rural se encuentre menor disponibilidad de servicios hace que las y los profesionales abarquen mayor número de casos de distintas áreas multiproblemáticas. Con los resultados obtenidos, observamos que las áreas donde surgen más cuestiones éticas son los referidos a: refugiados y asilados ( $87 \%$ ), familia ( $87 \%$ ), 
emigrantes $(69,6 \%)$, personas con discapacidades $(69,6 \%)$ y juventud $(66 \%)$. Es de subrayar que, a pesar de la tipificación de casos obtenida, el porcentaje de "otros grupos necesitados" alcanza un $60,9 \%$.

En este último porcentaje, cabe destacar la tipología de casos por la proporción que adquiere: 4,3\% varón autóctono; 5,1\% desempleado de larga duración; y $13,7 \%$ persona en riesgo o situación de exclusión social. El $16 \%$ restante se ciñe al ítem de "otros", sin que haya especificado ninguna respuesta.

En cuanto a la tarea que desempeñan las y los profesionales cuando les surge algún dilema ético, encontramos los siguientes resultados: área de trabajo principal como trabajador social (42,9\%), seguido de educador social (33,3\%), gestor $(9,5 \%)$ y nadie que se relacione como supervisor. Los últimos dos resultados obtenidos esclarecen la dualidad que perciben las y los profesionales, ya que muchos no veían distinción en la tarea que realizaban. Es por ello por lo que en el caso del supervisor nadie haya contestado, como también ocurre en el caso del gestor, que tiene un porcentaje mínimo (9,5\%). En resumen, existe transversalidad de tareas en el desempeño profesional de las y los trabajadores de lo social.

El problema más frecuente con el que se encuentran las y los profesionales de los servicios es el de la autonomía de la persona usuaria. Problema que requiere de una intervención más social y continua, un acompañamiento integral, más allá de ser "meros expendedores de recursos".

“Algunas o bastantes veces" las personas usuarias presentan: problemas económicos (71,4\%), racismo $(67 \%)$, alojamiento $(66,7 \%)$, conflictos familiares $(66,7 \%)$, desempleo (62\%), drogadicciones $(61,9 \%)$, alcoholismo $(61,9 \%)$ y violencia contra las mujeres $(57,1 \%)$. "Nunca o pocas veces”, los porcentajes más altos se encuentran en problemáticas de: violencia $(61,9 \%)$, prostitución $(61,9 \%)$, problemas de salud mental $(57,6 \%)$, vivienda $(57,2 \%)$ y ludopatías $(52,1 \%)$.

\subsection{Entrevistas}

A continuación se analizan las entrevistas realizadas. La posible causa de los valores mencionados en el apartado anterior puede tener que ver con la crisis padecida en España desde el 2007, que se ha plasmado en la cronificación de las situaciones de desempleo, como se corrobora en la siguiente entrevista:

[...] antes, antes de la crisis yo creo que se podría decir que el mayor factor de exclusión era el económico; eso es lo que marcaba, si no tenías un trabajo [...]. (Entrevista 5)

Sobre el racismo, que tiene un porcentaje del $67 \%$, una de las profesionales señala el cambio de perfil frente a los rumores extendidos tanto en la zona como dentro del colectivo de trabajadoras/es del servicio, que piensan que los extranjeros son los que requieren mayor cantidad de recursos:

[...] con prestaciones económicas, entonces cogí todas y dije "autóctonos inmigrantes”, una vez de tener todos esos datos yo pregunté a las compañeras a ver qué percepción teníamos, qué sensación teníamos. Y todas decíamos mucho más “inmigrantes y autóctonos" [...]. (Entrevista 5)

En el caso de problemáticas relacionadas con violencia contra las mujeres, cabe destacar una situación que generó un dilema ético, dado su carácter multidisciplinar:

[...] entonces eso es a nivel individual, pero es que luego se hace más difícil cuando tienes que trabajar un caso, por ejemplo, a nivel multidisciplinar. Porque, claro, para mí es una duda qué hacemos, denunciamos, no denunciamos, entonces a lo mejor para otro profesional que está actuando en el mismo caso dice que no, que tiene reservas, que nos dé su competencia etcétera, etcétera [...]. (Entrevista 7)

Aunque no sea un ítem presente en las encuestas realizadas, en la mayoría de las entrevistas ha aparecido una cuestión que genera un dilema ético en las y los profesionales, que es la cuestión de los traslados, tanto en los casos de problemas en salud mental, como en infancia, menores y familia. La razón de la problemática reside en dos cuestiones: la ubicación en una zona rural y el perfil de las personas usuarias. La primera razón se debe a que la mayoría de los servicios se encuentran centralizados en Pamplona y su comarca. De ahí que el traslado para la persona usuaria esté condicionado por este factor. Esto puede tener que ver con varios aspectos que se entrelazan en casos multiproblemáticos, como por ejemplo el coste económico y de espacio-tiempo que supone movilizarse desde el valle de Sakana hasta Pamplona y sus alrededores.

Por otro lado, la dimensión geográfica del valle y su orografía dejan algunos de los municipios más aislados que otros. Debido a ello, muchos de los pueblos no cuentan con transporte público. Frente a esta situación, las personas usuarias despliegan distintas estrategias que requieren de esfuerzos de diferente índole. Una de ellas dirigirse a un servicio privado como el taxi, lo cual puede generar otro tipo de gasto económico. Otra estrategia puede ser la de recurrir a familiares, vecinos, amistades del pueblo.

\section{Conclusiones y propuestas}

A raíz de los resultados obtenidos, en las próximas líneas se presentan las conclusiones y posteriormente se recopilan las propuestas realizadas por las y los profesionales. En primer lugar, existe una identificación consciente de los dilemas éticos en las entrevistas. Algo que no sucede en los cuestionarios, donde no los identifican. 
Acorde con investigaciones pioneras en ética de la intervención social en España, existe una variación en la identificación de los dilemas éticos en función del ámbito en el que las y los trabajadores desempeñen su labor profesional y de la estructura de la institución en la que se lleve a cabo (Viscarret, Ballestero, Idareta y Úriz, 2016).

Los problemas más habituales son los relacionados con la autonomía de la persona y los traslados. Las y los profesionales señalan el dilema que encuentran entre el principio de bienestar de la persona usuaria y la normativa existente. La normativa actual prohíbe que las y los profesionales trasladen a las personas usuarias independientemente del motivo que les haya llevado a tomar esa dicisión. No obstante, las propias particularidades de los casos muchas de las veces requieren el traslado, ya que por distintas razones (económicas, familiares, etc.) las personas usuarias no cuentan con los recursos y vías suficientes para realizarlo. La mayoría de los casos se debe a que los recursos y servicios se encuentran ubicados en Pamplona. Con un menor porcentaje (37\%), el estudio de Úriz, Ballestero y Urien (2007) también muestra que la mala distribución de los recursos genera dilemas éticos.

A los problemas más habituales se les suma la falta de formación continua en ética. De ahí el gran interés de las y los profesionales por subrayarlo, puesto que creen que hay una falta de lenguaje común a la hora de trabajar en red y en equipos multidisciplinares. Muchos de los profesionales mencionan los malentendidos que se ocasionan al trabajar en grupo por tener distinta comprensión del lenguaje técnico.

Un aspecto a destacar es que la mayoría de las y los trabajadores señalan que existe un desajuste entre la realidad de la zona rural y la normativa existente. Por eso, a la hora de resolver dilemas éticos, la mayoría recurre a las y los compañeros. Así lo corrobora también el estudio realizado por úriz, Ballestero y Urien (2007), donde los participantes, en la mayoría de los casos, tienden a recurrir a las y los compañeros más cercanos. Ninguno de los entrevistados ha recurrido a documentos escritos (por ejemplo, código deontológico) o a algún comité institucional de ética. De la misma manera que en el estudio de Úriz, Ballestero y Urien (2007), los y las profesionales con mayor experiencia no contemplan las teorías éticas o las consultas a la legislación, sino que confían más en la pericia adquirida en su trayectoria profesional.

Para finalizar, se exponen las propuestas realizadas por las profesionales. En primer lugar, manifiestan la necesidad de una formación continua en materia de ética, puesto que la que reciben en la actualidad les parece discontinua y limitada. Por eso reiteran la importancia de que la formación provenga desde la Administración Pública, ya que en algunos de los casos ha tenido que ser financiada por la y el profesional o el servicio donde desempeñan su labor.

En segundo lugar, señalan la necesidad de crear un grupo de supervisión con algún profesional externo como recurso de apoyo. En tercer y último lugar, ven indispensable un mayor apoyo de la Administración Pública que les ampare como servicios sociales de base de zonas rurales. Esto requiere una comunicación continua con las Administraciones Públicas y el traspaso de información pertinente de nuevos recursos, además de homogeneizar el lenguaje técnico entre las y los profesionales. 
AKINSOLA, H.A. (2001): "Ethical issues in rural nursing practice in Botswana", Nursing Ethics, vol. 8, n. $\stackrel{\circ}{4}$, pp. $340-349$.

ARANGUREN, N., PEÑA, L. y VILLAÑO, G. (2008): I. Primera jornada de Trabajo Social. Una mirada ética a la profesión, Vitoria, Gráficas Vicuña.

BALLESTERO, A., VISCARRET, J.J. y ÚRIZ, M.J. (2012): Dilemas éticos de las trabajadoras y los trabajadores sociales en España, Papers, vol. 97, n. $\stackrel{0}{4}$, pp. 875-898.

BANKS, S. (1997): Ética y valores en el trabajo social, Barcelona, Paidós.

BERMEJO, J. y DE LA RED, N. (1996): Ética y Trabajo Social, Madrid, Universidad Pontifica de Comillas.

CONSEJO GENERAL DEL TRABAJO SOCIAL -CGTS- (2012): Memoria. [/http://www.cgtrabajosocial.es/ app/webroot/files/consejo/files/memoria\%20 2012.pdf $>$ ]

CORTINA, A. y CONILL, J. (2000): 10 palabras clave en Ética de las Profesiones, Navarra, Verbo Divino.

DOMINGUÉZ, M.J. (2005): Cambios sociales actuales y adaptación de la profesión a los mismos, Humanismo y trabajo social, vol. $10, \mathrm{n} . \stackrel{0}{4}$, págs. $65-90$.

GARCíA, G. (1978): Los servicios sociales en el medio rural, Consejo General de Colegios Oficiales de Diplomados en Trabajo Social y Asistentes Sociales.

HERNÁNDEZ, J. (2013): Funciones de los profesionales de trabajo social en España, Cuadernos de Trabajo Social, vol. 21, n. $\stackrel{0}{1}$, págs. 127-138.

IDARETA, F. (2013): Teorías éticas en el Trabajo Social: Cuestionario de autoevaluación ética (C-AE), Trabajo social hoy, n. $\stackrel{6}{69}$, págs. 39-56.

IDARETA, F.; ÚRIZ, M.J.; y VISCARRET, J.J. (2017): 150 años de historia de la ética del Trabajo Social en
España: periodización de sus valores éticos, Cuadernos de Trabajo Social, vol. 30, n.ำ1, págs. 37-50.

MACKIE, P.F.E. (2007): Understanding the Educational and Demographic Differences between Rural and Urban Social Workers, The Journal of Baccalaureate Social Work, vol. 12, n.ํㅜㄹ, págs. 114-128.

NELSON, W.; GREENE, M.A.; y WEST, A. (2010): Rural healthcare ethics: No longer the forgotten quarter, Cambridge Quarterly of Healthcare Ethics, vol. 19, n. $\stackrel{0}{4}$, págs. 510-517.

NELSON, W.; POMERANTZ, A.; y WEEKS, W. (2006): Response to commentaries on «ls there a rural ethics literature?», American Journal of Bioethics, vol. 6 , n. $\stackrel{\circ}{4}$, págs. $46-47$.

PESUT, B.; BOTTORFF, J.L.; y ROBINSON, C.A. (2011): Be known, be available, be mutual: A qualitative ethical analysis of social values in rural palliative care, BMC Medical Ethics, vol. 12, ก. $\stackrel{o}{1}$.

ROBERTS, L.W.; BATTAGLIA, J.; y EPSTEIN, R.S. (1999): Frontier ethics: Mental health care needs and ethical dilemmas in rural communities, Psychiatric Services, vol. 50, n. $\stackrel{\circ}{4}$, págs. 497-503.

ROBERTS, L.W.; WARNER, T.D.; y HAMMOND, K.G. (2005): Ethical challenges of mental health clinicians in rural and frontier áreas, Psychiatric Services, vol. 56, n. $\stackrel{\circ}{3}$, págs. $358-359$.

ÚRIZ, Mạ.J.; BALLESTERO, A.; y URIEN, B. (2007): Dilemas éticos en la intervención social, España, Mira Editores.

VISCARRET, J.J., BALLESTERO, A., IDARETA, F. y ÚRIZ, M.J. (2016): Tipologías actuales de los trabajadores sociales en España, Cuadernos de Trabajo Social, vol. 29, n.ํㅜ 2, págs. 239-262. 